\title{
PHYSICS-BASED RETRIEVAL OF SURFACE ROUGHNESS PARAMETERS FOR BARE SOILS FROM COMBINED ACTIVE-PASSIVE MICROWAVE SIGNATURES
}

\author{
Anke Fluhrer', Thomas Jagdhuber ${ }^{l}$, Dara Entekhabi ${ }^{2}$, Michael H. Cosh ${ }^{3}$, Peggy O’Neill ${ }^{4}$, Roger Lang ${ }^{5}$, Ismail Baris ${ }^{l}$ \\ ${ }^{1}$ German Aerospace Center, Microwaves and Radar Institute, P. O. BOX 1116, 82234 Weßling, Germany. \\ ${ }^{2}$ Department of Civil and Environmental Engineering, Massachusetts Institute of Technology, Cambridge, MA, 02139 USA. \\ ${ }^{3}$ Hydrology and Remote Sensing Laboratory, United States Department of Agriculture/Agricultural Research Service (USDA-ARS), \\ Beltsville, MD 20705 USA. \\ ${ }^{4}$ Hydrological Science Laboratory, NASA Goddard Space Flight Center, Greenbelt, MD 20771 USA \\ ${ }^{5}$ Departement of Electrical \& Computer Engineering, George Washington University Washington, DC 20052 USA
}

\begin{abstract}
In the past the effect of soil roughness was often considered secondary within the determination of soil moisture from remote sensing data. Several studies showed that accurate determination of soil roughness leads to an improved estimation of soil moisture [1]. Two default parameters to describe the surface roughness are the standard deviation of the surface height variation $s$ and the surface correlation length $l$ with its corresponding autocorrelation function [2]. Both parameters $(s, l)$ affect the emissivity measured by radiometers as well as the backscattering observed by radars [1]. In this study, we develop a physics-based approach to retrieve $s$ and $l$ by combining both microwave signals based on active-passive microwave covariation. To test the approach, containing a forward model and a retrieval algorithm [3], [4], we used active/passive microwave data measured with the ComRAD truck-based SMAP simulator at L-band [5]. Results and validations with corresponding field measurements on ground show that $s$ and $l$ can be estimated simultaneously when using this approach. The physics-based retrieval algorithm works robustly for two investigated test fields having an RMS-Error of $0.68 \mathrm{~cm}$ and $0.69 \mathrm{~cm}$ between the microwave-based and field-measured $s$-values, and of $3.13 \mathrm{~cm}$ and $3.04 \mathrm{~cm}$ for $l$-values. The first validation of the results reveals that the influence of the autocorrelation function, needed within the retrieval, is distinct.
\end{abstract}

Index Terms - radar, radiometer, soil roughness, RMS height, correlation length, ComRAD

\section{INTRODUCTION}

Since soil moisture controls the water and energy exchange between pedosphere and atmosphere over continental regions, it is a key variable together with soil roughness and vegetation biomass that affects the emissivity $E[-]$ and the backscattering $\left|S_{P P}\right|^{2}[\mathrm{~dB}]$ characteristics of natural surfaces
[6]. The estimation of soil moisture through satellite sensor systems using combined radar and radiometer data has already been explored in several studies [7]. But until recently the effect of soil roughness on moisture retrieval was not in focus of research, albeit the precise determination of surface roughness leads to performant results for monitoring of soil moisture [1]. Since soil roughness strongly influences processes like infiltration, evaporation, soil erosion and growth of agricultural plants [8], we present in the following the theory and application of a new method to determine soil surface roughness through the combination of active and passive microwave signatures, whose results can then be used for instance in a consecutive step for a refined estimation of soil moisture. In order to determine the surface roughness parameters $s$ and $l$, we link active radar and passive radiometer microwave signatures at L-band through a linear functional relationship between land surface backscattering $\left(\left|S_{P P}\right|^{2}[\mathrm{~dB}]\right)$, and the respective natural microwave emission in form of brightness temperature $\left(T_{b P}\right.$ [K]) [9].

\section{TEST SITE AND DATA BASE}

The data base for this study are quasi-simultaneously acquired active and passive microwave measurements collected with the ComRAD (combined radar/radiometer) truck-based SMAP simulator, as well as in situ soil roughness data derived from digital photographs of the field surface profile against a known grid, collected during the APEX12 field experiment carried out by the National Aeronautics and Space Agency (NASA) and the U.S. Department of Agriculture (USDA) in Maryland 2012 [5], [6]. The active and passive microwave measurements were conducted under dry conditions with the ComRAD truckbased SMAP simulator at incident angle of $40^{\circ}$ over soybean (located south from the truck $=$ field south) and corn (located north from the truck $=$ field north) fields throughout the entire crop growth cycle from June to October 2012. The ComRAD system were mounted on a 
hydraulic boom truck in $19 \mathrm{~m}$ height between both fields. Results presented in this study assess only data acquired during the month June, since we delineate analyses with a novel physics-based model to calculate surface roughness parameters solely over bare soils. Detailed descriptions of technical properties of the ComRAD truck-based SMAP simulator as well as of the study site can be found in [5] and [6].

\section{METHODS}

In Figure 1 we defined several processing steps for the algorithm to determine the surface roughness parameters $S$ and $l$, which will be presented in detail in the following.

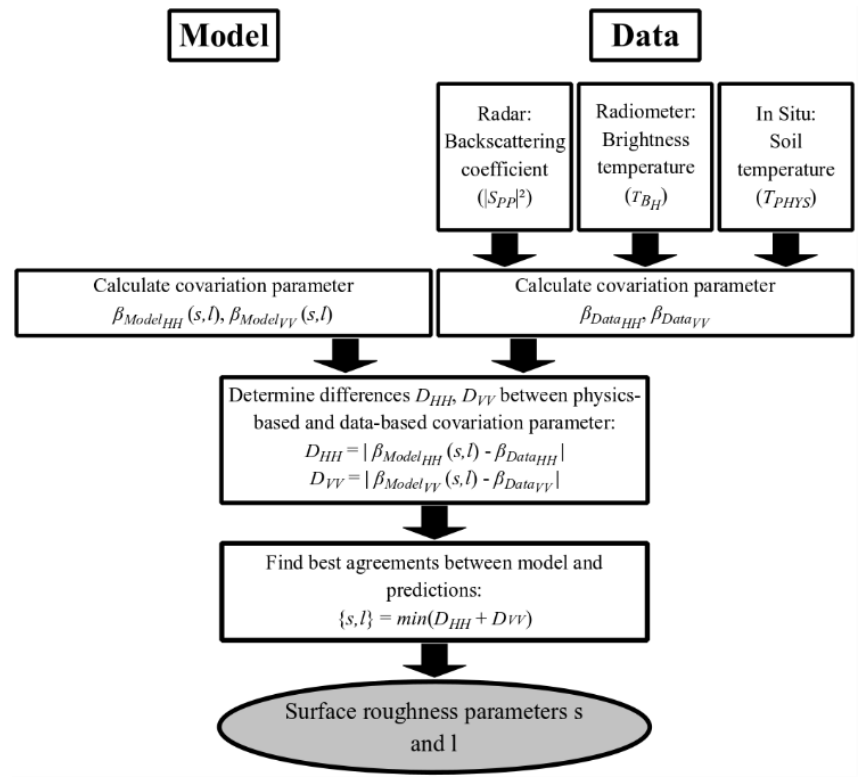

Figure 1: Workflow of the algorithm for determination of surface roughness parameters $s$ and $l$ derived from $p$ polarized differences between the physics-based model for covariation $\boldsymbol{\beta}_{\text {Model }_{P P}}$ and the data-retrieved covariation $\boldsymbol{\beta}_{\text {Data }_{P P}}$.

\subsection{Physics-based formulation for Active-Passive Microwave Covariation}

For joint evaluation of measurements from radar and radiometer sensors, their covariations with regard to soil moisture are one feasible option. Thereby, a physics-based formulation of covariation can be used based on Kirchhoff's law of energy conservation [3].

The inversion of this physics-based formulation leads to a data-based retrieval of active-passive microwave covariation [4]. The relationship between the backscattering coefficient $\left(\left|S_{P P}\right|^{2}[\mathrm{~dB}]\right)$ of the radar and the emission $(E[-])$ of the radiometer is functionally linear and can be represented by the two regression parameters $\alpha$ and $\beta$, with $\alpha$ being the intercept and $\beta$ being the slope of the linear regression [9]. For bare soils the intercept $\alpha$ is 1 , due to the fact that vegetation volume scattering does not occur [3]. Therefore, the slope $\beta$ describes the covariation between $E$ and $\left|S_{P P}\right|^{2}$ as a direct function of soil roughness for bare soils [3] and can be modelled as well as retrieved with the physics-based formulation. Hence, the covariation parameter $\beta$ can be calculated from quasi-simultaneously acquired active and passive microwave measurements [4].

Therefore, we forward model the covariation parameter $\beta$ and then retrieve it from data. Afterwards we determine the best fit between data and model predictions by minimizing their difference, in order to estimate the corresponding surface roughness parameters $s$ and $l$ (cf. Fig. 1).

\subsubsection{Definition of Forward Model}

The equation for the modeled covariation parameter $\beta_{\text {Model }_{P P} \text { [-] (cf. Fig. 1) for bare soils (no vegetation cover) }}$ sensing at L-band is the ratio of Fresnel $f_{F}$ and Bragg $f_{B}$ roughness loss terms $\beta_{\text {Model }_{H H}}=\frac{f_{F}}{f_{B}}$ [2], [10], which is additionally scaled by the conversion coefficient $\kappa_{\varepsilon}[-]$ for vertical polarization $\left(\beta_{\text {Model }_{V V}}=\frac{f_{F}}{f_{B} * \kappa_{\varepsilon}}\right)$ including the dielectric constant of soil $\varepsilon_{S}[-]$. However, sensitivity analyses results revealed that the influence of $\varepsilon_{s}$ on $\beta_{\text {Model }}$ is negligible within the proposed approach.

Calculations of the surface roughness parameters $s$ and $l$ can be done assuming different correlations between the surface height at one point and the surface height at another point (described by its autocorrelation function (ACF)) [4]. The Fresnel and Bragg roughness loss terms can include a Gaussian, exponential or power law ACF [11]. The Bragg roughness loss term in general is defined by $f_{B}=8 *$ $\left(\cos ^{2} \theta_{i} * k^{2} * s * l\right)^{2} * W(2 k \sin \theta)[10]$, with the wave number $k=\frac{2 \pi}{\lambda}$.

The Fourier transform of the surface correlation function $W(2 k \sin \theta)$ is defined as the height probability distribution function. Assuming a Gaussian ACF, it is given by [10]

$$
W(2 k \sin \theta)=\frac{l^{2}}{2} e^{\left(-(k * l * \sin \theta)^{2}\right)} .
$$

For an exponential ACF it is defined as [11]

$$
W(2 k \sin \theta)=l^{2} \frac{1}{\left(1+(2 \cdot k \cdot \sin \theta)^{2} \cdot l^{2}\right)^{\frac{3}{2}}} .
$$

For describing surface spectra $W(2 k \sin \theta)$ ranging between Gaussian and exponential types, the generalized power law spectrum for 2-D rough surfaces is used [11]

$$
W^{n}(2 k \sin \theta) \approx \frac{\frac{l}{n f p}^{2}}{2}(p-1) \frac{a_{p}^{2}}{b_{p}^{2}}\left[1+\frac{a_{p}^{2}}{b_{p}^{2}} \frac{(2 k \sin \theta)^{2}\left(\frac{l}{n f p}\right)^{2}}{4}\right]^{-p},
$$

with $f p=0.5 *\left[1+\left(\frac{1.5}{p}\right)^{2}\right], \quad a_{p}=\frac{\Gamma(p-0.5)}{\Gamma(p)} \quad$ and $\quad b_{p}=$ $1.17422(p-1.01793)(p-0.733964)^{-1}-0.176782$ [approximation with Mathematica ${ }^{\odot}$ similar to [12]]. Hereby applies that for $p=1$ the equation is equivalent to the exponential $\mathrm{ACF}$, and for $p=\infty$ the equation aligns to the Gaussian ACF. Hence, for $1<p>\infty$ equation (3) describes intermediate power law ACF types with $p$ as power 
coefficient [10], [11]. Calculations presented in this study delineate results with $p=1.75$ for field north and $p=$ 2.625 for field south, received from in situ roughness measurements. To conclude, the Bragg $f_{B}$ roughness loss term varies for different ACFs through the respective surface correlation function $(W(2 k \sin \theta))$.

In case of the Fresnel roughness loss term, $f_{F}$ varies only within the exponent $n$ for varying ACFs, which leads to $f_{F}=e^{-4\left(k * s * \cos \theta_{i}\right)^{n}}$. For Gaussian $n$ is 2 , for exponential $n$ is 1 , and for the power law ACF $n$ is adjusted to an adequate number between 1 and 2 [11]. For calculations presented in this study, we re-scaled the received power law values $p$ in order to determine appropriate $n$-values, leading to $n=1.0018$ for field north and $n=1.0026$ for field south.

Consequently, with the covariation parameter $\beta_{\text {Model }_{P P}}$ being the ratio of Fresnel $f_{F}$ and Bragg $f_{B}$ roughness loss terms, the equation for the Gaussian ACF, substituting the surface correlation function $W(2 k \sin \theta)$ with $(1)$, leads to:

$$
\beta_{\text {Model }_{\text {Gaus } V V}}=-\frac{f_{F}}{f_{B} * \kappa_{\varepsilon}}-\frac{e^{-4\left(k s * \cos \theta_{i}\right)^{n}}}{4 *\left(\cos ^{2} \theta_{i} * k^{2} * S * l\right)^{2} * e^{\left(-\left(k * l * \sin \theta_{i}\right)^{2}\right)} * \kappa_{\varepsilon}}
$$

Due to the fact, that for horizontal polarization $\kappa=1$ because of the equality of Fresnel and Bragg reflection coefficients [3], the equation hereby is:

$$
\beta_{\text {Model }_{\text {Gauss } H}}=-\frac{f_{F}}{f_{B}}=-\frac{e^{-4\left(k s * \cos \theta_{i}\right)^{n}}}{4 *\left(\cos ^{2} \theta_{i} * k^{2} * S * l\right)^{2} * e^{\left(-\left(k * l * \sin \theta_{i}\right)^{2}\right)}}
$$

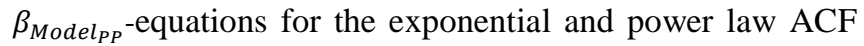
differ only in surface correlation function $W(2 k \sin \theta)$, by respectively substituting (2) or (3).

\subsubsection{Data-based Retrieval}

The equation for the data-based covariation parameter $\beta$, denoted as $\beta_{\text {Data }_{P P}}$ [-] for polarization $P$, follows according to [4], which represents the inversion of the physics-based formulation of [3]:

$$
\beta_{\text {Data }_{P P}}=\frac{E-1}{\left|S_{P P}\right|^{2}}=\frac{\frac{T_{b P}}{T_{p h y s}}-1}{\left|S_{P P}\right|^{2}}
$$

with $E$ being the emissivity, defined as ratio of the measured brightness temperature $T_{b P}[\mathrm{~K}]$ of the surface the physical temperature $\left(T_{\text {phys }}[\mathrm{K}]\right)$, and $\left|S_{P P}\right|^{2}[\mathrm{~dB}]$ being the measured normalized backscattering coefficient [4].

\subsection{Estimation of Surface Roughness Parameters $s$ and $l$}

In order to estimate the surface roughness parameters $s$ and $l$ we determined the best fit between model-based and databased covariation parameters $\beta$. Therefore, we calculate the differences $D_{P P}$ (7) between $\beta_{\text {Model }_{P P}}$ and $\beta_{\text {Data }_{P P}}$ for the horizontal and vertical polarizations (cf. Fig. 1).

$$
D_{P P}=\left|\beta_{\text {Model }_{P P}}(s, l)-\beta_{\text {Data }_{P P}}\right|
$$

The respective results for $D_{H H}$ and $D_{V V}$ for each data pixel are listed in a matrix with the dimension of the pre-defined ranges of roughness parameters $s$ and $l$ in the forward model. If we add up the matrices for both polarizations (8) we receive a look-up-table (LUT) where the number of columns represents the range of $s$ and the number of rows represents the range of $l$.

$$
\{s, l\}=\min \left(D_{H H}+D_{V V}\right)
$$

And the position of the smallest value in the LUT corresponds to the best-fitting values for $s$ and $l$.

\section{ROUGHNESS RETRIEVAL RESULTS}

In Figure 2 retrieval results for surface roughness parameters $s$ and $l$ are displayed exemplarily for field south including the three types of ACFs.
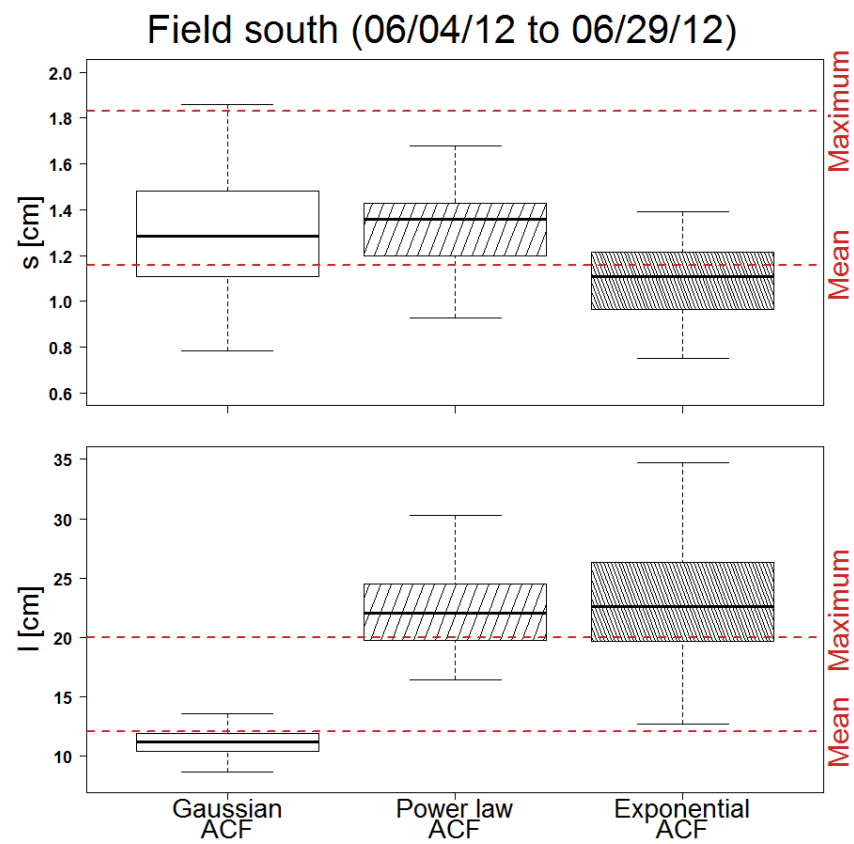

Figure 2: Retrieved surface roughness results for vertical RMS height $s$ (top) and horizontal length $l$ (bottom) over field south for three ACF types, in comparison with mean and maximum values of in situ measured surface roughness from the APEX12 field campaign (horizontal dashed lines).

In general, results for the surface roughness parameter $s$, calculated with a Gaussian ACF, are in the range from 0.75 to $1.94 \mathrm{~cm}$ over field north, and 0.78 to $2.32 \mathrm{~cm}$ over field south. Results of $s$ received for the exponential ACF are in the range from 0.86 to $1.36 \mathrm{~cm}$ over field north and 0.75 to $1.39 \mathrm{~cm}$ over field south. And finally, results for $s$ retrieved from the power law ACF are over field north between 0.79 to $1.39 \mathrm{~cm}$ and over field south between 0.93 to $1.68 \mathrm{~cm}$. Hence, in summary the lowest values for surface roughness parameter $s$ are reached with the exponential ACF, but overall results received with all ACFs are within a comparable range (cf. Fig. 2).

The situation is completely different for the horizontal correlation length $l$. As can be seen in Figure 2, only the 
Gaussian ACF delivers comparable results to in situ measured correlation length $l$.

This is confirmed by statistics with the RMS-Error being 3.13 and $3.09 \mathrm{~cm}$ for fields north and south, respectively. The RMS-Error between calculated and in situ measured correlation length $l$ assuming an exponential or power law $\mathrm{ACF}$ is $15 \mathrm{~cm}$ in average. Concluding, differences between field north and field south, which both have similar surface characteristics, are truly minor with the standard deviation over field north being $0.27(s)$ or $1.12(l)$, and over field south being $0.24(s)$ or $1.12(l)$.

\section{FIRST CONLCUSION}

In this study, we presented a physics-based approach to simultaneously determine surface roughness parameters $(s, l)$ from combined polarimetric radar and radiometer signatures. Results showed, that the approach leads to physically valid retrievals, and that validations with in situ measured roughness values indicate the potential of the combined active-passive retrieval. The results delineate a smooth surface with $s$ over field north being between 0.75 to 1.94 , and over field south being between 0.75 to 2.32 [2]. This is in line with observations made during the APEX12 experiment.

\section{REFERENCES}

[1] Saatchi, S., Njoku, E., Wegmüller, U. (1993). "Synergism of active and passive microwave data for estimating bare soil surface moisture", ESA/NASA Int. Workshop Passive Microwave Remote Sens. Res. Related to Land-atmosphere. Interactions, Saint-Lary, France, Jan. 1115, pp. 205-224.

[2] Ulaby, F.T., Long, D.G., Blackwell, W., Elachi, Ch., Fung, A., Ruf, C., Sarabandi, K., Zebker, H., van Zyl, J.J. (2014). "Microwave radar and radiometric remote sensing", University of Michigan. Press Ann Arbor, 2014.

[3] Jagdhuber, T., Koning, A.G., McColl, K.A:, Alemohammad, S.H., Das, N.N., Montzka, C., Link, M., Akbar, R., Entekhabi, D. (2017). "Physics-Based Modeling of Active and Passive Microwave Covariations Over Vegetated Surfaces", in: IEEE Transactions on geoscience and remote sensing (In Review).

[4] Jagdhuber, T., Entekhabi, D., Konings, A.G., McColl, K.A., Alemohammad, S.H., Das, N.N., Montzka, C., Piles, M. (2016). "Physically-based retrieval of SMAP activepassive measurements covariation and vegetation structure parameters", in: International Geoscience and Remote Sensing Symposium (IGARSS), Vol. 2016-11-1, pp. 30783081, Conference Paper.

[5] O’Neill, P. (2012). "Soil Moisture active passive mission (SMAP) - ComRAD Active/Passive L-band Instrument System." 3rd Cal/Val Workshop Nov. 14-16, 2012.
[6] Srivastava, P., O'Neill, P., Cosh, M. H., Kurum, M., Lang, R., Joseph, A. (2014). "Evaluation of Dielectric Mixing Models for Passive Microwave Soil Moisture Retrieval Using Data From ComRAD Ground-Based SMAP Simulator", Selected Topics in Applied Earth Observations and Remote Sensing, IEEE. vol. 99: 1-10.

[7] Wu, X., Walker, J.P., Rüdiger, C., Panciera, R. (2015). "Effect of Land-Cover Type on the SMAP Active/Passive Soil Moisture Downscaling Algorithm Performance", in: IEEE Geoscience and remote sensing letters, vol. 12, no. 4.

[8] Marzahn, P., Ludwig, R. (2009). "On the derivation of soil surface roughness from multi parametric PolSAR data and its potential for hydrological modeling", Hydrol. Earth Syst. Sci., 13, 381-394.

[9] Das, N. N., Entekhabi, D., Njoku, E. G., Shi, J. J. C., Johnson, J. T., Colliander, A. (2014). "Tests of the SMAP Combined Radar and Radiometer Algorithm Using Airborne Field Campaign Observations and Simulated Data", in: IEEE Transactions on Geoscience and Remote Sensing, vol. 52, no. 4, pp. 2018-2028

[10] Ulaby, F. T., Moore, R. K., \& Fung, A. K. (1982). "Microwave remote sensing: Active and passive. Volume 2Radar remote sensing and surface scattering and emission theory." Boston, USA, Artech House.

[11] Li, Q., Shi, J.-C., Chen, K.S. (2002). “A Generalized Power Law Spectrum and its Applications to the Backscattering of Soil Surfaces Based on the Integral Equation Model", in: IEEE Transactions on Geoscience and Remote Sensing, vol. 40, pp. 517-526.

[12] Loew, A., Mauser, W. (2006). "A semi empirical surface backscattering model for bare soil surfaces based on a generalized power law spectrum approach", in IEEE Transactions on Geoscience and Remote Sensing, vol. 44, no. 4, pp. 1022-1035. 